\title{
Discontinuous Galerkin Methods for Elliptic problems
}

\author{
Douglas N. Arnold ${ }^{1}$, Franco Brezzi ${ }^{2}$, Bernardo Cockburn ${ }^{3}$, and Donatella \\ Marini $^{2}$ \\ 1 Department of Mathematics, Penn State University, University Park, PA 16802, \\ USA \\ 2 Dipartimento di Matematica and I.A.N.-C.N.R. Via Ferrata 1, 27100 Pavia, \\ Italy \\ 3 School of Mathematics, University of Minnesota, Minneapolis, Minnesota \\ 55455 , USA
}

\begin{abstract}
We provide a common framework for the understanding, comparison, and analysis of several discontinuous Galerkin methods that have been proposed for the numerical treatment of elliptic problems. This class includes the recently introduced methods of Bassi and Rebay (together with the variants proposed by Brezzi, Manzini, Marini, Pietra and Russo), the local discontinuous Galerkin methods of Cockburn and Shu, and the method of Baumann and Oden. It also includes the so-called interior penalty methods developed some time ago by Douglas and Dupont, Wheeler, Baker, and Arnold among others.
\end{abstract}

\section{Introduction}

In 1973, Reed and Hill [21] introduced the first discontinuous Galerkin (DG) method for hyperbolic equations, and since that time there has been an active development of DG methods for hyperbolic and nearly hyperbolic problems, resulting in a variety of different methods. Also in the 1970's, but independently, Galerkin methods for elliptic and parabolic equations using discontinuous finite elements were proposed, and a number of variants introduced and studied. These were generally called interior penalty (IP) methods and their development remained independent of the development of the DG methods for hyperbolic equations. In this paper, we provide a common framework which includes nearly all the DG methods that have been proposed thus far.

We briefly review the development of penalty methods for elliptic and parabolic equations. Penalties were first introduced into the finite element method as a mean for imposing Dirichlet boundary conditions weakly rather than incorporating the boundary conditions into the finite element space. Let us begin by recalling Nitsche's method [19] for the model problem $-\Delta u=f$ in $\Omega, u=0$ on $\partial \Omega$. Clearly

$$
\int_{\Omega} \nabla u \cdot \nabla v d x-\int_{\partial \Omega} \frac{\partial u}{\partial n} v d s=\int_{\Omega} f v d x
$$


for all sufficiently smooth test functions $v$. Since $u$ vanishes on the boundary, we have as well that $B(u, v)=\int f v d x$, where

$$
B(u, v):=\int_{\Omega} \nabla u \cdot \nabla v d x-\int_{\partial \Omega} \frac{\partial u}{\partial n} v d s-\int_{\partial \Omega} \frac{\partial v}{\partial n} u d s+\int_{\partial \Omega} \eta u v d s,
$$

for any weighting function $\eta$. Nitsche's method then determines an approximate solution $u_{h}$ in a finite element subspace of $H^{1}(\Omega)$ such that $B\left(u_{h}, v_{h}\right)=$ $\int f v_{h} d x$ for all $v_{h}$ in the same space. Note that the second term of the bilinear form $B$ arose to ensure that the method is consistent. The third term was added so that the discrete problem is symmetric (and so the method is truly variational - the discrete solution minimizes $B(u, u) / 2-\int f u$ over the finite element space). Finally, the last term is the penalty term, which is necessary to guarantee stability. Nitsche proved that if $\eta$ is taken as $C / h$ where $h$ is the element size and $C$ is a sufficiently large constant, then the discrete solution converges to the exact solution with optimal order in $H^{1}$ and $L^{2}$.

A different penalty method for imposing Dirichlet boundary conditions is due to Babuška [2]. He does not include either the second or third term in (1.1), and uses as the penalty weight $h^{-\sigma}$ for some $\sigma \geq 0$. Because of the missing consistency term, his method, and its analysis, includes a consistency error. Another interesting possibility is to include all the terms in (1.1) but to reverse the sign of the third term in $B$. The bilinear form is then no longer symmetric, but it has a favorable coercivity property, namely, $B(u, u) \geq$ $\int|\nabla u|^{2}$, no matter how $\eta \geq 0$ is chosen.

The IP methods arose from the observation that, just as Dirichlet boundary conditions could be imposed weakly instead of being built into the finite element space, so interelement continuity could be attained in a similar fashion. This makes it possible to use spaces of discontinuous piecewise polynomials for solving second order problems. The natural generalization of Nitsche's method to this context (in which there are consistency, symmetrization, and penalty terms on each edge, the latter penalizing the jump of the function across the edge) is stated in Wheeler's 1978 paper on IP collocation-finite element methods, [27], where it is attributed to a private communication of Douglas and Dupont. That method is analyzed in detail for linear and nonlinear elliptic and parabolic problems in the 1979 thesis of Arnold which is summarized in [1]. Interior penalties of this sort were also used by Baker [4] for imposing $C^{1}$ interelement continuity on $C^{0}$ elements for fourth order problems. In these, of course, it is the jump in the normal derivative that is penalized. In 1976, Douglas and Dupont [16] penalized the jump in the normal derivative of $C^{0}$ elements for second order elliptic and parabolic problems, with the goal of enforcing a degree of continuity in some sense intermediate between $C^{0}$ and $C^{1}$. Babuška and Zlámal [3], like Baker, used interior penalties to weakly impose $C^{1}$ continuity for fourth order problems, but their bilinear form is analogous to Babuška's finite element with penalty rather than to the bilinear form of Nitsche's method, i.e., it does not have the consistency and symmetry terms. 
Not so much attention has been paid to IP methods since the early 1980's, although they have found a few new applications. In 1990, Baker, Jureidini and Karakashian [5] used interior penalties to enforce continuity on piecewise solenoidal vector fields for solving the Stokes equations. In the same year, Rusten, Vassilevski, and Winther [23] used an interior penalty method for second order elliptic problems as part of a preconditioner for mixed methods. Recently, Becker and Hansbo [10] used the IP approach as a way to enforce continuity across non-matching grids for domain decomposition.

On the other hand, DG methods for the numerical treatment of nonlinear hyperbolic systems experienced a vigorous development during the last ten years due to a strong interaction with the ideas of finite volumes methods for hyperbolic problems; see a review of this development in [14]. But the evolution of the DG methods did not stop there. The necessity of dealing with problems that, together with a dominant convective part, had a non-negligible diffusive part, prompted several authors to extend the DG methods to elliptic problems. Thus in 1997, Bassi and Rebay [6] introduced a DG method for the Navier-Stokes equations and in 1998, Cockburn and Shu [15] introduced the so-called local discontinuous Galerkin (LDG) methods by generalizing the original DG method of Bassi and Rebay. Around the same time, Oden and Bauman [8], [9] introduced another DG method for diffusion problems. Their approach uses a non-symmetric bilinear form, even for symmetric problems, analogous to the one obtained from Nitsche's penalty form by reversing the sign of the symmetrization term, as discussed earlier.

It was at this point that several authors were struck by the similarities between those recently introduced DG methods and the old IP methods and started to apply to the former the old techniques of analysis used on the latter. Thus, Brezzi et al. [12] studied several variations of the original method of Bassi and Rebay; Oden, Babuška and Baumann [20] studied the DG method of Baumann and Oden; Rivière and Wheeler [22], [26] analyzed several variations of the DG method of Baumann and Oden; and Süli, Schwab, and Houston [24], [25] synthesized the elliptic, parabolic, and hyperbolic theory by extending the analysis of DG methods to partial differential equations with non-negative characteristic form. Our long term goal is to follow this trend and produce a comprehensive study of the above mentioned methods as applied to elliptic problems. In this note, we recast all of the above mentioned methods within a single framework in order to lay down a basis for a better understanding of the connections among them, and, eventually, a unified analysis, that however we postpone to a subsequent paper.

An outline of the paper is as follows. For the sake of simplicity and clarity, we present our unified framework for the classical problem of the Laplacian with homogeneous Dirichlet boundary conditions. In $\S 2$, we provide a general framework for its discretisation by means of DG methods. This framework is an extension of the approach used by Cockburn and Shu [15] to define the LDG methods and allows us to include methods that are not LDG methods, like the IP methods and the DG method of Baumann and Oden. In the 
next two sections we verify that indeed many known methods fall within our framework, and we present a partial classification of methods. A table listing all these methods is included in the final section.

\section{The general DG method for a model problem}

For the sake of simplicity, we restrict ourselves to the following model problem:

$$
-\Delta u=f \quad \text { in } \Omega, \quad u=0 \quad \text { on } \partial \Omega,
$$

where $\Omega$ is assumed to be a polygonal domain and $f$ a given function in $L^{2}(\Omega)$. To obtain the weak formulation upon which the discretization is based, we rewrite the above problem as follows:

$$
\sigma=\nabla u, \quad-\nabla \cdot \sigma=f \quad \text { in } \Omega, \quad u=0 \quad \text { on } \partial \Omega .
$$

Let $K$ be the closure of an open subset of $\Omega$ with a piecewise smooth boundary. If we multiply the above equations by test functions and integrate formally on $K$, we get

$$
\begin{aligned}
& \int_{K} \sigma \cdot \tau d x=-\int_{K} u \nabla \cdot \tau d x+\int_{\partial K} u n_{K} \cdot \tau d s, \\
& \int_{K} \sigma \cdot \nabla v d x=\int_{K} f v d x+\int_{\partial K} \sigma \cdot n_{K} v d s,
\end{aligned}
$$

where $n_{K}$ is the outward normal unit vector to $\partial K$. This is the weak formulation we sought. We are now ready to define the DG method.

We denote by $\mathcal{T}_{h}$ a triangulation of $\Omega$ in polygons $K$, and by $P(K)$ a finite dimensional space of smooth functions, typically polynomials, defined on the polygon $K$. This space will be used to approximate the variable $u$. We denote by $\Sigma(K)$ another finite dimensional space of smooth functions that we are going to use in order to approximate the auxiliary variable $\sigma$. Setting

$$
\begin{aligned}
& V_{h}:=\left\{v \in L^{2}(\Omega) \quad|\quad v|_{K} \in P(K) \quad \forall K \in \mathcal{T}_{h}\right\}, \\
& \Sigma_{h}:=\left\{\tau \in\left(L^{2}(\Omega)\right)^{2} \quad|\quad \tau|_{K} \in \Sigma(K) \quad \forall K \in \mathcal{T}_{h}\right\},
\end{aligned}
$$

and following Cockburn and Shu [15], we consider the following general weak formulation: Find $u_{h} \in V_{h}$ and $\sigma_{h} \in \Sigma_{h}$ such that $\forall K \in \mathcal{T}_{h}$ we have

$$
\begin{array}{ll}
\int_{K} \sigma_{h} \cdot \tau d x=-\int_{K} u_{h} \nabla \cdot \tau d x+\sum_{e \subset \partial K} \int_{e} h_{u}^{e, K} n_{K} \cdot \tau d s & \forall \tau \in \Sigma(K), \\
\int_{K} \sigma_{h} \cdot \nabla v d x=\int_{K} f v d x+\sum_{e \subset \partial K} \int_{e} h_{\sigma}^{e, K} \cdot n_{K} v d s & \forall v \in P(K),
\end{array}
$$


where the sums are taken over the edges $e$ of the polygon $K$, and the $n u$ merical fluxes $h_{\sigma}^{e, K}$ and $h_{u}^{e, K}$ are approximations to $\left.\sigma\right|_{e}=\left.\nabla u\right|_{e}$ and to $\left.u\right|_{e}$, respectively, on the edges of the triangulation. In order to complete the definition of a method we must provide the polynomial spaces $P(K)$ and $\Sigma(K)$ and the formula for the numerical fluxes $h_{\sigma}^{e, K}$ and $h_{u}^{e, K}$ in terms of $\sigma_{h}$ and $u_{h}$. The choice of spaces will not play a large role in our study. For triangular elements, one could, for example, take $P(K)$ to consist of all polynomials of degree $p \geq 1$ and $\Sigma(K)$ to consist of all polynomial vector fields of degree $p-1$ or $p$. The choice of the consititutive relations defining the fluxes, on the other hand, will be crucial. The flux choices affect the stability and the accuracy of the method, as well as properties such as sparsity and symmetry of the stiffness matrix; cf. [15] and [13]. As we shall see, different choices for the fluxes will lead to the different methods that we are going to discuss.

Next, we discuss some basic properties that are shared by all the flux choices.

1. Locality. Let $K=K_{1}$ be an element in the triangulation, and let $e$ be one of its edges. Assume first that $e$ is an interior edge of our triangulation, so that there is a second element $K_{2}$ sharing the edge $e$ with $K_{1}$. We then assume that $h_{\sigma}^{e, K}$ and $h_{u}^{e, K}$ depend on the restrictions $\left.u_{h}\right|_{K_{i}}$ and $\left.\sigma_{h}\right|_{K_{i}}$ of $u_{h}$ and $\sigma_{h}$ to $K_{i}, i=1,2$. More precisely, locality means that

$$
h_{\sigma}^{e, K}=h_{\sigma}^{e, K}\left(\left.u_{h}\right|_{K_{1}},\left.\sigma_{h}\right|_{K_{1}},\left.u_{h}\right|_{K_{2}},\left.\sigma_{h}\right|_{K_{2}}\right) .
$$

Actually, in all our examples, this fucntional dependence will have a special form in that both $h_{\sigma}^{e, K}$ and $h_{u}^{e, K}$ will depend only on the traces of $\left.u_{h}\right|_{K_{i}}$, $\left.\nabla u_{h}\right|_{K_{i}}$, and $\left.\sigma_{h}\right|_{K_{i}}$ on the edge $e$. Since $u_{h}, \nabla u_{h}$, and $\sigma_{h}$ will, in general, be discontinuous across $e$, the trace of $\left.u_{h}\right|_{K_{1}}$ on $e$ will be different from the trace of $\left.u_{h}\right|_{K_{2}}$ on $e$, and similarly $\nabla u_{h}$ and $\sigma_{h}$ will each have two different traces on $e$. Thus $h_{\sigma}^{e, K}$ and $h_{u}^{e, K}$ will depend linearly on the six quantities

$$
\left.\left(\left.u_{h}\right|_{K_{1}}\right)\right|_{e},\left.\left(\left.\nabla u_{h}\right|_{K_{1}}\right)\right|_{e},\left.\left(\left.\sigma_{h}\right|_{K_{1}}\right)\right|_{e},\left.\left(\left.u_{h}\right|_{K_{2}}\right)\right|_{e},\left.\left(\left.\nabla u_{h}\right|_{K_{2}}\right)\right|_{e},\left.\left(\left.\sigma_{h}\right|_{K_{2}}\right)\right|_{e} .
$$

In our particular case of a homogeneous Dirichlet problem, the fluxes on boundary edges will have the same functional dependence on these six traces, provided we interpret the traces coming from $K_{2}$ as follows: $\left.\left(\left.u_{h}\right|_{K_{2}}\right)\right|_{e}=$ $0,\left.\left(\left.\nabla u_{h}\right|_{K_{2}}\right)\right|_{e}=\left.\left(\left.\nabla u_{h}\right|_{K_{1}}\right)\right|_{e}$, and $\left.\left(\left.\sigma_{h}\right|_{K_{2}}\right)\right|_{e}=\left.\left(\left.\sigma_{h}\right|_{K_{1}}\right)\right|_{e}$. Other boundary conditions can be handled easily as well, but, in order to keep the notation as simple as possible, we shall not discuss these here.

Finally, it is important to note that in all the methods we are going to analyze, $h_{u}^{e, K}$ will not depend on $\left.\sigma_{h}\right|_{K_{i}}$ (nor on $\left.\nabla u_{h}\right|_{K_{i}}$, but that will be less important). This, as we shall see, will allow us to eliminate the variable $\sigma_{h}$ at the element level, often with a considerable computational saving.

2. Consistency. All the methods we consider are consistent in the sense that, in the functional form described above,

$$
\begin{aligned}
& h_{\sigma}^{e, K}\left(\left.u\right|_{K_{1}},\left.\nabla u\right|_{K_{1}},\left.u\right|_{K_{2}},\left.\nabla u\right|_{K_{2}}\right)=\left.\nabla u\right|_{e}, \\
& h_{u}^{e, K}\left(\left.u\right|_{K_{1}},\left.\nabla u\right|_{K_{1}},\left.u\right|_{K_{2}},\left.\nabla u\right|_{K_{2}}\right)=\left.u\right|_{e},
\end{aligned}
$$


whenever $u$ is a smooth function satisfying the boundary conditions.

3. Conservation. All our methods satisfy

$$
h_{\sigma}^{e, K_{1}}=h_{\sigma}^{e, K_{2}}
$$

when $e$ is an edge shared by elements $K_{1}$ and $K_{2}$, and so we may write simply $h_{\sigma}^{e}$. This is a conservation property: if $S$ is the union of some collection of elements, then, taking $v$ to be identically unity in (2.2) and adding over $K$ contained in $S$ we get

$$
\int_{S} f d x+\sum_{e \subset \partial S} \int_{e} h_{\sigma}^{e} \cdot n d s=0
$$

We close this section with several additional remarks concerning the above properties.

1. As we have seen, if $h_{u}^{e, K}$ does not depend on $\sigma_{h}$, then the auxiliary variable $\sigma_{h}$ can be eliminated locally in terms of $u_{h}$ and $\nabla u_{h}$, using (2.1). When using triangles, the use of the orthonormal Dubiner basis [17] renders this elimination trivial. See also the extensions to 3D elements by Lomtev and Karniadakis [18].

2. In all the methods we consider, $h_{\sigma}^{e}$ depends either on the traces of $\nabla u_{h}$ or on those of $\sigma_{h}$, but not on both. The former category, for which the stiffness matrix tends to be much sparser, includes the IP methods and the method of Baumann and Oden; we discuss this category of methods in $\S 4$. The latter category, which we discuss in $\S 3$, includes the LDG family of methods.

3. Most of the methods will satisfy, in addition to the conservation property (2.3), the analogous property $h_{u}^{e, K_{1}}=h_{u}^{e, K_{2}}$ (in which case we write $h_{u}^{e}$ for $h_{u}^{e, K}$.) We shall refer to them as completely conservative methods. As we shall see, generally only completely conservative methods lead to a symmetric stiffness matrix after elimination of $\sigma_{h}$. Except for the methods of Baumann and Oden, and the so-called pure penalty methods discussed at the end of $\S 4$, all the methods we consider are completely conservative.

4. We also note that, in view of $(2.2)$, only the normal component $h_{\sigma}^{e, K} \cdot n_{K}$ of $h_{\sigma}^{e, K}$ enters the methods; its tangential component is irrelevant. In practice, the normal component will depend only on the normal traces.

\section{Numerical fluxes independent of $\nabla u_{h}$}

In order to describe the flux functions for various methods we need to introduce some notation. Again let $e$ be an edge shared by elements $K_{1}$ and $K_{2}$. Define also the normal vectors $n_{1}$ and $n_{2}$ on $e$ pointing exterior to $K_{1}$ and 
$K_{2}$, respectively. If $v$ is a function on $K_{1} \cup K_{2}$, but possibly discontinuous across $e$, let $v_{i}$ denote $\left.\left(\left.v\right|_{K_{i}}\right)\right|_{e}, i=1,2$. For a scalar function $v$ we then define

$$
\{v\}:=\frac{1}{2}\left(v_{1}+v_{2}\right), \quad \llbracket v \rrbracket:=v_{1} n_{1}+v_{2} n_{2} .
$$

If $\tau$ is a vector-valued function, we set

$$
\{\tau\}:=\frac{1}{2}\left(\tau_{1}+\tau_{2}\right), \quad \llbracket \tau \rrbracket:=\tau_{1} \cdot n_{1}+\tau_{2} \cdot n_{2} .
$$

Notice that the jump $\llbracket v \rrbracket$ of the scalar function $v$ is a vector parallel to $n$ and that $\llbracket \tau \rrbracket$ is the jump of the normal component of the vector function $\tau$ - it is hence is a scalar quantity. The advantage of these definitions is that they do not depend on assigning an ordering to the elements $K_{i}$.

In this section, we consider the DG methods determined by the following choice of numerical fluxes:

$$
h_{\sigma}^{e, K}=\left\{\sigma_{h}\right\}-\alpha^{e}\left(\llbracket u_{h} \rrbracket\right)+\beta^{e} \llbracket \sigma_{h} \rrbracket, \quad h_{u}^{e, K}=\left\{u_{h}\right\}+\gamma^{e} \cdot \llbracket u_{h} \rrbracket .
$$

Here $\beta^{e}$ and $\gamma^{e}$ are vector-valued functions on $e$. Often they are constant, and, indeed, for many methods they both vanish. The term $\alpha^{e}\left(\llbracket u_{h} \rrbracket\right)$ could simply be taken to be

$$
\alpha^{e}\left(\llbracket u_{h} \rrbracket\right)=\eta^{e} \llbracket u_{h} \rrbracket
$$

for some constant (or function) $\eta^{e}$. Another possibility arises from the work of Bassi and Rebay. Namely we define the operator $r_{e}: L^{1}(e) \rightarrow \Sigma_{h}$ by

$$
\int_{\Omega} r_{e}(q) \cdot \tau d x=-\int_{e} q \cdot\{\tau\} d s \quad \forall \tau \in \Sigma_{h}, q \in L^{1}(e),
$$

and set

$$
\alpha^{e}\left(\llbracket u_{h} \rrbracket\right)=\eta^{e}\left\{r_{e}\left(\llbracket u_{h} \rrbracket\right)\right\} .
$$

First we rewrite the method by inserting the flux formulas (3.1) into the Galerkin equations (2.1)-(2.2) and adding over $K \in \mathcal{T}_{h}$. Denoting by $\mathcal{E}_{h}$ the set of all element edges, after simple algebraic manipulations we obtain

$$
\begin{aligned}
& \int_{\Omega} \sigma_{h} \cdot \tau d x=\sum_{K} \int_{K} \nabla u_{h} \cdot \tau d x+\sum_{e \in \mathcal{E}_{h}} \int_{e}\left(\gamma^{e} \cdot \llbracket u_{h} \rrbracket \llbracket \tau \rrbracket-\llbracket u_{h} \rrbracket \cdot\{\tau\}\right) d s, \\
& \sum_{K} \int_{K} \sigma_{h} \cdot \nabla v d x=\int_{\Omega} f v d x+\sum_{e \in \mathcal{E}_{h}} \int_{e}\left(\left\{\sigma_{h}\right\}-\alpha^{e}\left(\llbracket u_{h} \rrbracket\right)+\beta^{e} \llbracket \sigma_{h} \rrbracket\right) \cdot \llbracket v \rrbracket d s
\end{aligned}
$$

for all $\tau \in \Sigma_{h}, v \in V_{h}$. If we take all the $\alpha^{e}, \beta^{e}$, and $\gamma^{e}$ to vanish, we recover the original DG method of Bassi and Rebay, cf. [6], formulae (13) and (15), 
and also [11], equations (12) and (14). This method can be unstable, at least for uniform meshes; see [11]. However, stability is achieved if $\alpha^{e}$ is a positive operator. Defining $\alpha^{e}$ by (3.3) with $\eta^{e}>0$ (and still with $\beta^{e}$ and $\gamma^{e}$ zero) gives the variant of the method of Bassi and Rebay [6], as proposed by Brezzi et al. [12], formula (24). Defining $\alpha^{e}$ by (3.2), $\eta^{e}>0$ gives the LDG methods (which allow general $\beta^{e}$ and $\gamma^{e}$ ).

Next, we eliminate $\sigma_{h}$ to rewrite the method in terms of $u_{h}$ alone (this is usually the preferred implementation in practice). To do so, we define two operators, $R$ and $L$. The operator $R: V_{h} \rightarrow \Sigma_{h}$ is given by $R(v)=$ $\sum_{e \in \mathcal{E}_{h}} r_{e}(\llbracket v \rrbracket)$, or, equivalently,

$$
\int_{\Omega} R(\varphi) \cdot \tau d x=-\sum_{e \in \mathcal{E}_{h}} \int_{e} \llbracket \varphi \rrbracket\{\tau\} d s \quad \forall \tau \in \Sigma_{h}
$$

The operator $L: L^{1}\left(\bigcup \mathcal{E}_{h}\right) \rightarrow \Sigma_{h}$ is given by

$$
\int_{\Omega} L(\varphi) \cdot \tau d x=\sum_{e \in \mathcal{E}_{h}} \int_{e} \varphi \llbracket \tau \rrbracket d s \quad \forall \tau \in \Sigma_{h}
$$

Denoting by $P_{\Sigma}$ the $\mathrm{L}^{2}$-projection onto $\Sigma_{h}$, we can now rewrite equation (3.4) as

$$
\sigma_{h}=P_{\Sigma}\left(\nabla u_{h}\right)+R\left(u_{h}\right)+L\left(\gamma \cdot \llbracket u_{h} \rrbracket\right),
$$

and equation (3.5) as

$$
\begin{aligned}
\sum_{K} \int_{K} \sigma_{h} \cdot \nabla v d x=\int_{\Omega} f v d x+\int_{\Omega} \sigma_{h} \cdot(-R(v)+L(\beta \cdot \llbracket v \rrbracket)) \\
-\sum_{e \in \mathcal{E}_{h}} \int_{e} \alpha^{e}\left(\llbracket u_{h} \rrbracket\right) \cdot \llbracket v \rrbracket d s .
\end{aligned}
$$

Here we mean by $\beta$ and $\gamma$ the functions on $\bigcup \mathcal{E}_{h}$ which are given by $\beta^{e}$ and $\gamma^{e}$, respectively, on each edge $e$. Finally, inserting (3.8) in (3.9), we get

$$
\begin{aligned}
\sum_{K} \int_{K}\left(P_{\Sigma}\left(\nabla u_{h}\right)+R\left(u_{h}\right)\right. & \left.+L\left(\gamma \cdot \llbracket u_{h} \rrbracket\right)\right) \cdot(\nabla v+R(v)-L(\beta \cdot \llbracket v \rrbracket)) d x \\
& +\sum_{e \in \mathcal{E}_{h}} \int_{e} \alpha^{e}\left(\llbracket u_{h} \rrbracket\right) \cdot \llbracket v \rrbracket d s=\int_{\Omega} f v d x .
\end{aligned}
$$


Note that the second sum on the left-hand side of (3.10) is symmetric with respect to $u_{h}$ and $v$. Indeed

$$
\begin{aligned}
\sum_{e \in \mathcal{E}_{h}} \int_{e} \alpha^{e}\left(\llbracket u_{h} \rrbracket\right) \cdot \llbracket v \rrbracket d s & \\
= & \begin{cases}\sum_{e \in \mathcal{E}_{h}} \int_{e} \eta^{e} \llbracket u_{h} \rrbracket \cdot \llbracket v \rrbracket d s, & \text { if } \alpha^{e} \text { is defined by (3.2), } \\
\sum_{e \in \mathcal{E}_{h}} \int_{\Omega} \eta^{e} r_{e}\left(\llbracket u_{h} \rrbracket\right) \cdot r_{e}(\llbracket v \rrbracket) d x, & \text { if } \alpha^{e} \text { is defined by (3.3). }\end{cases}
\end{aligned}
$$

It is thus clear that a symmetric stiffness matrix is obtained if we choose $\beta^{e}=-\gamma^{e}$ for all $e$. This choice was suggested by Cockburn and Shu [15] for the LDG methods.

In practice the inclusion $\nabla P(K) \subset \Sigma(K)$ generally holds. In that case the projection $P_{\Sigma}$ is not needed in (3.10).

Finally, we remark that if the support of $v$ is contained in a single element $K$, then the support of $R(v)$ will generally contain all the elements that contain an edge of $K$. Consequently the product $R\left(u_{h}\right) \cdot R(v)$ in (3.10) will generally have a big negative impact on the sparsity of the stiffness matrix. This problem is much less severe when the numerical fluxes are independent of $\sigma_{h}$.

\section{Numerical fluxes independent of $\sigma_{h}$}

First we consider, instead of (3.1), the following numerical fluxes:

$$
h_{\sigma}^{e, K}=\left\{\nabla u_{h}\right\}-\alpha^{e}\left(\llbracket u_{h} \rrbracket\right)+\beta^{e} \llbracket \nabla u_{h} \rrbracket, \quad h_{u}^{e, K}=\left\{u_{h}\right\}+\gamma^{e} \llbracket u_{h} \rrbracket,
$$

where $\beta^{e}$ and $\gamma^{e}$ are still vector-valued functions on $e$. Let us proceed now to the elimination of the variable $\sigma_{h}$ as we did at the end of the previous section. By using the definitions of $R$ and $L,(3.6)$ and (3.7), respectively, a simple computation gives us that

$$
\begin{aligned}
\sum_{K} \int_{K}\left(P_{\Sigma}\left(\nabla u_{h}\right)\right. & \left.+R\left(u_{h}\right)+L\left(\gamma \cdot \llbracket u_{h} \rrbracket\right)\right) \cdot \nabla v \\
& +\nabla u_{h} \cdot(R(v)-L(\beta \cdot \llbracket v \rrbracket)) d x \\
& +\sum_{e \in \mathcal{E}_{h}} \int_{e} \alpha^{e}\left(\llbracket u_{h} \rrbracket\right) \cdot \llbracket v \rrbracket d x=\int_{\Omega} f v d x .
\end{aligned}
$$

For $\beta=\gamma=0$ and $\alpha$ chosen as in (3.2), we recover the old IP method of [16] and [1], while for $\beta=\gamma=0$ and $\alpha$ as in (3.3) we recover the second formulation of the DG method of Bassi and Rebay, see [7]. As proven in [11], under rather general assumptions, and for triangular elements, the scheme 
is stable and optimally convergent whenever $\eta^{e}>3$, where the number 3 represents, in essence, the number of edges per element.

Notice that now the number of non-zero entries of the stiffness matrix is reduced to its minimum. This is due to the fact that the term $R\left(u_{h}\right) \cdot R(v)$ that appeared in (3.10) is not present anymore in (4.1).

We now consider another family of numerical fluxes. Let us choose:

$$
h_{\sigma}^{e}=\zeta\left\{\nabla u_{h}\right\}-\alpha^{e}\left(\llbracket u_{h} \rrbracket\right), \quad h_{u}^{e, K}=\left\{u_{h}\right\}+\delta \llbracket u_{h} \rrbracket \cdot n_{K},
$$

where $\zeta$ and $\delta$ are real parameters. Different choices for these parameters will select different methods. We point out immediately that for $\delta \neq 0$ the corresponding methods will not be completely conservative, and for $\zeta \neq 1$ consistency will be violated.

Using (4.2) in (2.1)-(2.2) and proceeding in the elimination of $\sigma_{h}$ as before, we get

$$
\begin{aligned}
\sum_{K} \int_{K}( & \left.P_{\Sigma}\left(\nabla u_{h}\right) \cdot \nabla v+(1-2 \delta) R\left(u_{h}\right) \cdot \nabla v+\zeta \nabla u_{h} \cdot R(v)\right) d x \\
& +\sum_{e \in \mathcal{E}_{h}} \int_{e} \alpha^{e}\left(\llbracket u_{h} \rrbracket\right) \cdot \llbracket v \rrbracket d x=\int_{\Omega} f v d x .
\end{aligned}
$$

For $\delta=1, \zeta=1, \alpha^{e}=0$, and $\nabla P(K) \subset \Sigma(K)$ (so restricted to $\nabla P(K)$, $P_{\Sigma}$ reduces to the inclusion operator and can be suppressed), this is exactly the DG method of Baumann and Oden. To see this, let us rewrite the above equation. We start by noting that

$$
\int_{\Omega} \nabla u \cdot R(v) d x=-\sum_{e \in \mathcal{E}_{h}} \int_{e} \llbracket v \rrbracket\{\nabla u\} d s=-\sum_{K} \int_{\partial K}(v v \mid) \frac{\partial u}{\partial n_{K}} d s,
$$

where we set, in each element $K$, for every $e \in \partial K$,

$$
(v \mid)=\frac{1}{2}\left(v^{i n t}-v^{e x t}\right)_{e},
$$

with obvious meaning of the symbols. With this notation and when $\nabla P(K) \subset$ $\Sigma(K)$, the equation (4.3) can be rewritten as

$$
\begin{gathered}
\sum_{K}\left(\int_{K} \nabla u_{h} \cdot \nabla v d x+\int_{\partial K}\left((2 \delta-1)\left(\left|u_{h}\right|\right) \frac{\partial v}{\partial n}-\zeta(v \mid) \frac{\partial u_{h}}{\partial n}\right) d s\right) \\
+\sum_{e \in \mathcal{E}_{h}} \int_{e} \alpha^{e}\left(\llbracket u_{h} \rrbracket\right) \cdot \llbracket v \rrbracket d s=\int_{\Omega} f v d x,
\end{gathered}
$$

which is nothing but the DG method of Baumann and Oden when $\delta=\zeta=1$ and $\alpha^{e}=0$, as claimed. This scheme has been analyzed by Oden, Babuška, and Baumann [20], and requires some extra assumptions to achieve stability, e.g., polynomials of degree $\geq 2$. The situation would clearly improve by taking 
$\alpha^{e}$ as in (3.2) or (3.3) with $\eta^{e}>0$. This is also indicated by Süli, Schwab, and Houston in [24] and [25], where a full analysis of these methods (with $\delta=0$ or $1, \zeta=1$ and $\alpha^{e}$ as in (3.2), $\left.\eta^{e}>0\right)$ is performed.

On the other hand, by taking $\delta=1 / 2$ and $\zeta=0$ in (4.2), equation (4.3) becomes

$$
\sum_{K} \int_{K} P_{\Sigma}\left(\nabla u_{h}\right) \cdot \nabla v d x+\sum_{e \in \mathcal{E}_{h}} \int_{e} \alpha^{e}\left(\llbracket u_{h} \rrbracket\right) \cdot \llbracket v \rrbracket d x=\int_{\Omega} f v d x .
$$

This, when $\nabla P(K) \subset \Sigma(K)$, can be seen as an extension of the BabuškaZlámal IP method [3] to second order elliptic problems, when $\alpha^{e}$ is chosen as in (3.2). If instead, $\alpha^{e}$ is chosen as in (3.3), we obtain the penalty formulation proposed in [12]. Note that both methods are inconsistent, so that, in both cases, $\eta^{e}$ has to go to $+\infty$ when the meshsize tends to zero, although with different speed for the two cases; for triangular grids, $\eta^{e}$ should behave as $|e|^{-2 p-1}$ in the former case, and as $|e|^{-2 p}$ in the latter, where $p$ is the degree of the polynomials in $P(K)$.

\section{Concluding remarks}

In this paper, we have proposed a unified framework to study a large class of DG methods for elliptic problems. This class includes the classical IP methods as well as practically all the recently introduced DG methods. The following table summarizes the flux choices needed to obtain the methods discussed; for all these methods $P(K)$ is a standard polynomial space and $\Sigma(K)$ is taken large enough to contain $\nabla P(K)$.

\begin{tabular}{lcc}
\hline Method & $h_{\sigma}^{e, K}$ & $h_{u}^{e, K}$ \\
\hline Bassi-Rebay 1 & $\left\{\sigma_{h}\right\}$ & $\left\{u_{h}\right\}$ \\
Brezzi et al. 1 & $\left\{\sigma_{h}\right\}-\eta^{e}\left\{r_{e}\left(\llbracket u_{h} \rrbracket\right)\right\}$ & $\left\{u_{h}\right\}$ \\
LDG & $\left\{\sigma_{h}\right\}-\eta^{e} \llbracket u_{h} \rrbracket+\beta^{e} \llbracket \sigma_{h} \rrbracket$ & $\left\{u_{h}\right\}+\gamma^{e} \llbracket u_{h} \rrbracket$ \\
IP & $\left\{\nabla u_{h}\right\}-\eta^{e} \llbracket u_{h} \rrbracket$ & $\left\{u_{h}\right\}$ \\
Bassi-Rebay 2 & $\left\{\nabla u_{h}\right\}-\eta^{e}\left\{r_{e}\left(\llbracket u_{h} \rrbracket\right)\right\}$ & $\left\{u_{h}\right\}$ \\
Baumann-Oden & $\left\{\nabla u_{h}\right\}$ & $\left\{u_{h}\right\}-\llbracket u_{h} \rrbracket \cdot n_{K}$ \\
Babuška-Zlámal & $-\eta^{e} \llbracket u_{h} \rrbracket$ & $\left.u_{h}\right|_{K}$ \\
Brezzi et al. 2 & $-\eta^{e}\left\{r_{e}\left(\llbracket u_{h} \rrbracket\right)\right\}$ & $\left.u_{h}\right|_{K}$ \\
\hline
\end{tabular}


We saw that this class subdivides naturally into completely conservative methods and partially conservative methods, on the one hand, and into methods whose fluxes are independent of $\sigma_{h}$ and methods who aren't. We saw that completely conservative methods give rise to symmetric problems when the parameters of their numerical fluxes are suitably defined, and that partially conservative methods might give rise to non-symmetric methods. We also saw that DG methods whose numerical fluxes are independent of $\sigma_{h}$ produce stiffness matrices with a remarkably smaller number of non-zero entries.

We believe that such a unified framework could facilitate the understanding of the various methods and their relationships, as well as a possible unified analysis of their convergence properties.

\section{References}

1. D.N. Arnold, An interior penalty finite element method with discontinuous elements, SIAM J. Numer. Anal. 19 (1982), 742-760.

2. I. Babuška, The finite element method with penalty, Math. Comp. 27 (1973), 221-228.

3. I. Babuška and M. Zlámal, Nonconforming elements in the finite element method with penalty, SIAM J. Numer. Anal. 10 (1973), 863-875.

4. G.A. Baker, Finite element methods for elliptic equations using nonconforming elements, Math. Comp. 31 (1977), 45-59.

5. G.A. Baker, W.N. Jureidini, and O.A. Karakashian, Piecewise solenoidal vector fields and the Stokes problem, SIAM J. Numer. Anal. 27 (1990), 1466-1485.

6. F. Bassi and S. Rebay, A high-order accurate discontinuous finite element method for the numerical solution of the compressible Navier-Stokes equations, J. Comput. Phys. 131 (1997), 267-279.

7. F. Bassi, S. Rebay, M. Savini, G. Mariotti, and S. Pedinotti, A high-order accurate discontinuous finite element method for inviscid and viscous turbomachinery flows, Proceedings of the Second European Conference on Turbomachinery, Fluid Dynamics and Thermodynamics, March 5-7, 1997, Antwerpen, Belgium.

8. C.E. Baumann and J.T. Oden, A discontinuous hp finite element method for convection-diffusion problems, Comput. Methods Appl. Mech. Engrg., in press, special issue on Spectral, Spectral Element, and $h p$ Methods in CFD, edited by G.E. Karniadakis, M. Ainsworth and C. Bernardi.

9. __ A discontinuous hp finite element method for the Navier-Stokes equations, 10th. International Conference on Finite Element in Fluids, 1998.

10. R. Becker and P. Hansbo, A finite element method for domain decomposition with non-matching grids, Tech. Report 3613, INRIA, 1999.

11. F. Brezzi, D. Marini, P. Pietra, and A. Russo, Discontinuous finite elements for diffusion problems, Atti Convegno in onore di F. Brioschi, Istituto Lombardo, Accademia di Scienze e Lettere, 1997, to appear.

12. __ Discontinuous finite elements for diffusion problems, Numerical Methods for Partial Differential Equations (1999), submitted.

13. P. Castillo, An optimal error estimate for the local discontinuous Galerkin method, this volume, pp. - .

14. B. Cockburn, G.E. Karniadakis, and C.-W. Shu, The evolution of discontinuous Galerkin methods, this volume, pp. -. 
15. B. Cockburn and C.W. Shu, The local discontinuous Galerkin finite element method for convection-diffusion systems, SIAM J. Numer. Anal. 35 (1998), 2440-2463.

16. J. Douglas, Jr. and T. Dupont, Interior penalty procedures for elliptic and parabolic Galerkin methods, Lecture Notes in Physics, vol. 58, Springer-Verlag, Berlin, 1976.

17. M. Dubiner, Spectral methods on triangles and other domains, J. Sci. Comp. 6 (1991), 345.

18. I. Lomtev and G.E. Karniadakis, A discontinuous Galerkin method for the Navier-Stokes equations, Int. J. Numer. Meth. Fluids 29 (1999), 587-603.

19. J.A. Nitsche, Über ein Variationsprinzip zur Lösung Dirichlet-Problemen bei Verwendung von Teilräumen, die keinen Randbedingungen unteworfen sind, Abh. Math. Sem. Univ. Hamburg 36 (1971), 9-15.

20. J.T. Oden, Ivo Babuška, and C.E. Baumann, A discontinuous hp finite element method for diffusion problems, J. Comput. Phys. 146 (1998), 491-519.

21. W.H. Reed and T.R. Hill, Triangular mesh methods for the neutron transport equation, Tech. Report LA-UR-73-479, Los Alamos Scientific Laboratory, 1973.

22. B. Rivière and M.F. Wheeler, Part I. Improved energy estimates for interior penalty, constrained and discontinuous Galerkin methods for elliptic problems, Tech. Report 99-09, TICAM, 1999.

23. T. Rusten, P.S. Vassilevski, and R. Winther, Interior penalty preconditioners for mixed finite element approximations of elliptic problems, Math. Comp. 65 (1996), 447-466.

24. E. Süli, Ch. Schwab, and P. Houston, hp-DGFEM for partial differential equations with non-negative characteristic form, this volume, (http://www.comlab.ox.ac.uk/oucl/publications/natr/NA-99-02.html), pp. - .

25. E. Süli and Ch. Schwab and P. Houston, hp-finite element methods for hyperbolic problems, The Mathematics of Finite Elements and Applications, MAFELAP X (J. R. Whiteman, ed.), Springer Verlag, June 1999, to appear (http://www.comlab.ox.ac.uk/oucl/publications/natr/NA-99-11.html).

26. M.F. Wheeler, this volume, pp. - .

27. , An elliptic collocation-finite element method with interior penalties, SIAM J. Numer. Anal. 15 (1978), 152-161. 